\title{
Generalized Casimir forces in nonequilibrium systems
}

\author{
R. Brito, ${ }^{1}$ U. Marini Bettolo Marconi, ${ }^{2}$ and R. Soto ${ }^{3}$ \\ ${ }^{1}$ Departamento de Física Aplicada I and GISC, Universidad Complutense, 28040 Madrid, Spain \\ ${ }^{2}$ Dipartimento di Fisica, Università di Camerino, 62032 Camerino, Italy \\ ${ }^{3}$ Departamento de Física, FCFM, Universidad de Chile, Casilla 487-3, Santiago, Chile \\ (Received 13 February 2007; revised manuscript received 11 May 2007; published 19 July 2007)
}

\begin{abstract}
In the present work, we propose a method to determine fluctuation-induced forces in nonequilibrium systems. These forces are the analog of the well-known Casimir forces, which were originally introduced in quantum field theory and later extended to the area of critical phenomena. The procedure starts from the observation that many nonequilibrium systems exhibit fluctuations with macroscopic correlation lengths, and the associated structure factors strongly depend on the wave vectors for long wavelengths; in some cases the correlations become long range, and the structure factors show algebraic divergences in the long-wavelength limit. The introduction of external bodies into such systems in general modifies the spectrum of these fluctuations, changing the value of the renormalized pressure, which becomes inhomogeneous. This inhomogeneous pressure leads to the appearance of a net force between the external bodies. It is shown that the force can be obtained from the knowledge of the structure factor of the homogeneous system. The mechanism is illustrated by means of a simple example: a reaction-diffusion equation, where the correlation function has a characteristic length. The role of this length in the Casimir force is elucidated.
\end{abstract}

DOI: 10.1103/PhysRevE.76.011113

PACS number(s): 05.40.- a, 05.20.Jj, 74.40.+k

\section{INTRODUCTION}

In 1948, Casimir [1] noticed that, since in the vacuum the quantum electromagnetic field fluctuates rather than vanishing as in the classical case, it would cost some energy to introduce a macroscopic body into it. Indeed, the presence of a macroscopic body limits the allowed wave vectors and therefore the energy density. Casimir derived a simple expression for the resulting force per unit area acting between two large conducting neutral plates facing each other at distance $x$. The result is $F / A=-\hbar c \pi^{2} /\left(240 x^{4}\right)$, where the presence of $\hbar c$ reveals the quantal origin of the force. In the last 20 years, Casimir's idea has been extended to some equilibrium systems where fluctuations are of thermal origin, rather than quantal, and thus the force is proportional to $k_{B} T$ [2]. The unifying feature of systems displaying thermal Casimir forces is the presence of long-ranged spatial correlations. The best-known cases are binary fluid mixtures at the critical point [3] and other critical systems [4-6], where the Casimir force between two plates of area $A$ and separation $x$ is attractive and proportional to $-k_{B} T A / x^{3}$. The inverse power law character of the force is originated by the divergence of the correlation length $\xi$ as the critical point is approached. The Ornstein-Zernike theory [4] predicts long-range density fluctuations characterized by a structure factor (defined as the Fourier transform of the density-density correlation function) like $S(\mathbf{k}) \propto 1 /\left(\xi^{-2}+k^{2}\right)$, where $\xi \propto\left|T-T_{c}\right|^{-1 / 2}$. Then the structure factor behaves as $S(k) \propto k^{-2}$ at the critical point, and the free energy of the system scales as $k_{B} T A / x^{d-1}$, from which one derives the above expression for the force.

Casimir forces also appear in equilibrium physical systems where long-range correlations appear because a continuous symmetry is broken [7]. For instance, in liquid crystals, with a vector order parameter, the structure factor for a smectic- $A$ phase layered in the $z$-direction is anisotropic: $S(\mathbf{k}) \propto k_{\perp}^{2} /\left(k_{z}^{2}+\lambda^{2} k_{\perp}^{4}\right)$. When the $\mathbf{k}$ is parallel to the layers, i.e., $k_{z}=0$, the structure factor diverges as $k^{-2}$. Such smectic liquid crystals also exhibit a Casimir force: $F \propto-k_{B} T A / x^{2}$ [8]. Similar long-ranged correlations appear also in nematic phases of liquid crystals, or magnetic liquid crystals immersed in a magnetic field $H$ [9], and in superfluid films [10], and in solutions of ionic salts or micellar systems [11], among many others.

The first approach to treating thermal Casimir forces closely follows the method originally proposed by Casimir in the quantum electrodynamic case: one computes the sizedependent free energy and then obtains the force by differentiating it with respect to distance [2]. Also, Casimir forces can be understood in terms of a stress tensor that is size dependent, due to the long-range correlations. It has been possible to compute the Casimir force as the difference of the size-dependent pressure between a confined and an infinite system, using the scale invariance property of critical fluids $[5,12]$, scale-free nonequilibrium systems [13], and critical spin models [14].

These approaches, however, do not stress enough the relation between long-range correlations and Casimir forces. In particular, the case where there is no scale invariance and there is a finite correlation length has not been considered.

The goal of this paper is to establish a general relation between correlation functions (or the associated structure factors) and Casimir forces. We provide a method to compute the size-dependent pressure and the Casimir force, especially suitable for nonequilibrium systems, where longrange correlations are ubiquitous [15], while arguments based on the free energy cannot be invoked. It is shown that the Casimir force can be obtained from the knowledge of the structure factor of the homogeneous system.

There are numerous nonequilibrium systems for which the structure factors have been obtained. A major class is represented by systems under spatial gradients, such as an isothermal uniformly sheared fluid in the $z$ direction (plane Couette flow) $[16,17]$. It was found that the structure factor 
in the limit $k \rightarrow 0$ behaves as $S(\mathbf{k}) \propto-\dot{\gamma}_{0} k_{x} k_{z} / k^{4}$, where $\dot{\gamma}_{0}$ is the shear rate. In a similar fashion, a fluid in a RayleighBenard cell, with a temperature gradient $\nabla T$ below the onset of the instability, shows a correlation function with a $1 / k^{2}$ dependence [18]. In a binary mixture under the same experimental conditions, there is a nonlinear enhancement of the structure factors proportional to $(\nabla T)^{2} / k^{4}$. Here the fluctuations are measured in the $x y$ plane, orthogonal to the gradient of the temperature $[19,20]$. In the same Rayleigh-Benard cell, but heated from above, Li et al. showed that the concentration fluctuations in a polymer solution behave as $(\nabla c)^{2} / k^{4}$, where $\nabla c$ is the concentration gradient created by the Soret effect. Pure concentration gradients were studied by Spohn [21] using lattice gases; that author showed that $S(k) \propto(\nabla c)^{2} /\left(k^{2}+k_{0}^{2}\right)$, with $k_{0}=\pi /(2 L), L$ being the system size.

Another class of nonequilibrium systems exhibiting longrange correlations are those lacking the detailed balance condition. For instance, it has been shown $[22,23]$ that systems described by the Langevin equation with conservative dynamics and nonconservative noise have long-range spatial and temporal correlations. Analogously, nonequilibrium concentration fluctuations in reaction-diffusion systems can be long ranged under certain conditions [24] that will be described in this paper.

There are a few cases of nonequilibrium systems with long-range correlations where a Casimir force has been derived, for example, the already mentioned scale-free nonequilibrium systems [13], a driven one-dimensional system [25], and a granular fluid [26]. In the granular case, the balance between the energy dissipation due to collisions and the energy injection renders the system statistically stationary, but out of equilibrium. As a consequence, it can develop long-range correlations, and the structure factors for the hydrodynamic fields decay as $k^{-2}$ [27].

\section{THE MESOSCOPIC MODEL}

As an example of how a Casimir force arises in a nonequilibrium system with long-range correlations, we consider the simple case of a reaction-diffusion system in three dimensions, where the fluctuating density $n$ around the homogeneous reference density $n_{0}$ obeys the equation

$$
\frac{\partial \phi}{\partial t}=\boldsymbol{\nabla} \cdot\left(D \boldsymbol{\nabla} \phi+\boldsymbol{\xi}_{1}\right)-\lambda \phi+\xi_{2},
$$

where $\phi=n-n_{0}$ is the fluctuating field, $D$ is the diffusion coefficient, and $\lambda>0$ is the relaxation rate. The terms $\boldsymbol{\xi}_{1}$ and $\xi_{2}$ describe fluctuations in the diffusive flux and in the reaction rate, respectively. They are assumed to have white noise spectra

$$
\begin{aligned}
\left\langle\xi_{1 i}(\mathbf{r}, t) \xi_{1 k}\left(\mathbf{r}^{\prime}, t^{\prime}\right)\right\rangle & =\Gamma_{1} \delta_{i, k} \delta\left(\mathbf{r}-\mathbf{r}^{\prime}\right) \delta\left(t-t^{\prime}\right), \\
\left\langle\xi_{2}(\mathbf{r}, t) \xi_{2}\left(\mathbf{r}^{\prime}, t^{\prime}\right)\right\rangle & =\Gamma_{2} \delta\left(\mathbf{r}-\mathbf{r}^{\prime}\right) \delta\left(t-t^{\prime}\right),
\end{aligned}
$$

where $\Gamma_{1}$ and $\Gamma_{2}$ are the noise intensities. The noises $\xi_{1}$ and $\xi_{2}$ are referred to, respectively, as conservative and nonconservative noises because the former conserves the total mass, while the latter makes it fluctuate.
This reaction-diffusion equation models, for instance, the $k_{1}$ set of chemical reactions $A \rightarrow B, A+B \rightarrow 2 A$, which do not satisfy the detailed balance condition [28]. In this case, $\phi$ is the density fluctuation of either species $A$ or $B$ relative to its average stationary density, and $\lambda=n_{T} k_{2}-k_{1}$, with $n_{T}$ the total density. When $\lambda=0$ the reaction shows a critical point at $n_{T}$ $=k_{1} / k_{2}$. We assume that the pressure in the system is a function of the local density $p(n)$.

Equation (1) not only models the chemical reaction described above, but governs many other physical systems, like liquid crystals [29] or superfluid films [2]. All these systems possess structure factors similar to those mentioned in the Introduction, and the results obtained in this paper can be generalized to those systems.

The solution of Eq. (1) predicts that, after an initial transient, the density $\phi$ is statistically homogeneous and stationary, so that in Fourier space we have

$$
\left\langle\phi_{\mathbf{k}}\right\rangle=0, \quad\left\langle\phi_{\mathbf{k}} \phi_{\mathbf{q}}\right\rangle=V S(\mathbf{k}) \delta_{\mathbf{k},-\mathbf{q}},
$$

where the symbol $\langle\cdot\rangle$ represents the average over the two noises $\xi_{1}$ and $\xi_{2}$. The structure factor $S(\mathbf{k})$ and its Fourier transform, the space correlation $G(\mathbf{r})$, are given by (see [24], Chap. 8.3)

$$
\begin{gathered}
S(\mathbf{k})=\frac{\Gamma_{1}}{2 D}+\frac{\Gamma / 2 D}{k^{2}+k_{0}^{2}}, \\
G(\mathbf{r})=\frac{\Gamma_{1}}{2 D} \delta(\mathbf{r})+\frac{\Gamma}{2 D} \frac{e^{-k_{0} r}}{r},
\end{gathered}
$$

with $\Gamma=\Gamma_{2}-\Gamma_{1} \lambda / D$ and $k_{0}=\sqrt{\lambda / D}$. The second contribution in $G(\mathbf{r})$, stemming from the $k$-dependent term of $S(\mathbf{k})$, represents fluctuations with a correlation length that depends on the parameters $D$ and $\lambda$, and, therefore, is of macroscopic size. In particular, near the critical point $\lambda \rightarrow 0$, the correlation length diverges. If the reaction satisfies the fluctuationdissipation theorem $[24,30]$, then $\Gamma_{1}=2 k_{B} T D$ and $\Gamma_{2}$ $=2 k_{B} T \lambda$, where $T$ is the temperature, implying that $\Gamma$ vanishes along with the macroscopic correlations. On the contrary, in nonequilibrium systems which violate the fluctuation-dissipation theorem, $\Gamma$ does not vanish and macroscopic correlations are present. The $\delta$ term of $G(\mathbf{r})$ in Eq. (5), coming from $\Gamma_{1} / 2 D$, describes the microscopic selfcorrelation of the particles that a mesoscopic model, valid for larger length scales, cannot resolve. These correlations are present in both equilibrium and nonequilibrium, and, as will become manifest in the next paragraphs, they do not contribute to the Casimir forces. Therefore, they will be eliminated henceforth. This corresponds to subtracting the asymptotic value of $S$ for large values of $k$. Hereafter, we will consider the macroscopic part (or, equivalently, the nonequilibrium part) of the structure factor

$$
S^{*}(\mathbf{k})=S(\mathbf{k})-\lim _{q \rightarrow \infty} S(q)=\frac{\Gamma}{2 D\left(k^{2}+k_{0}^{2}\right)} .
$$

This is equivalent to suppressing the vectorial (conserved) noise $\boldsymbol{\xi}_{1}$ and keep only a scalar (nonconserved) noise $\xi$ with an intensity $\Gamma$. 


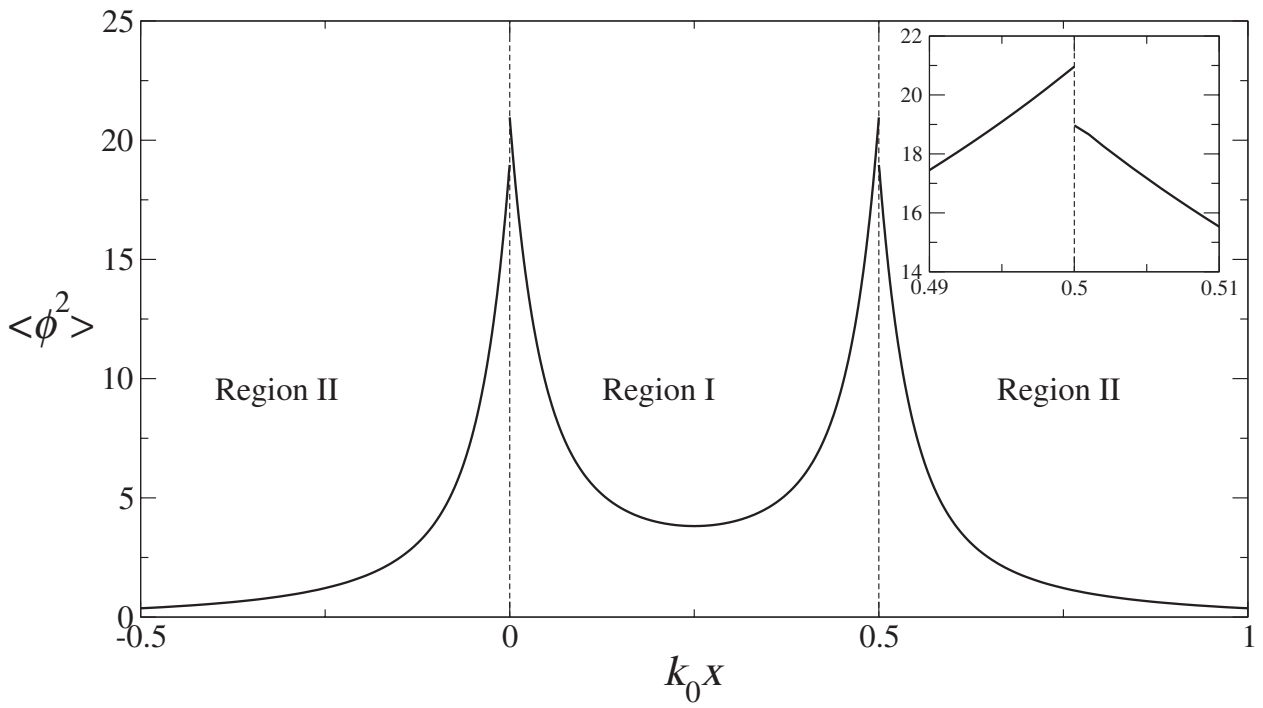

FIG. 1. Density fluctuations as a function of the dimensionless position $k_{0} x$, for $\epsilon=0.05$ and $k_{0} L$ $=1 / 2$. The vertical scale is in arbitrary units and the asymptotic value of the density fluctuations has been subtracted. The inset shows the density fluctuation jump at the plate.
We study, now, the effect of confining the system between two plates, parallel and infinite in the $y$ and $z$ directions, located at $x=0$ and $x=L$, and ask whether the confinement of the fluctuating field can produce a Casimir force between the plates surrounded by a fluid described by Eq. (1). The force derives from the pressure, which we shall assume to be a local function of the density, i.e., $p(n)$, exerted by the particles over the plates. To proceed, we consider the system in a volume $L_{x} \times L_{y} \times L_{z}$, periodic in all directions. In this volume we place two plates at distance $L$ with nonflux boundary conditions at them, as natural for a reacting system. The total volume $V$ is divided into two regions: region I between the plates, of volume $L \times L_{y} \times L_{z}$, and region II, outside the plates, of volume $\left(L_{x}-L\right) \times L_{y} \times L_{z}$. The limit $L_{x}, L_{y}, L_{z} \rightarrow \infty$ will eventually be taken.

In order to perform the analysis in the two regions, let us consider the case of a general volume $V=X \times L_{y} \times L_{z}$, where $X=L$ for region I and $X=L_{x}-L$ for region II. The density field is expanded, taking into account the nonflux boundary conditions in the $x$ direction, as

$$
\phi(\mathbf{r}, t)=V^{-1} \sum_{k_{x}} \sum_{k_{y}} \sum_{k_{z}} \phi_{\mathbf{k}}(t) \cos \left(k_{x} x\right) e^{i k_{y} y} e^{i k_{z} z},
$$

where $k_{x}=\pi n_{x} / X, k_{y}=2 \pi n_{y} / L_{y}, k_{z}=2 \pi n_{z} / L_{z}, n_{x}=0,1,2, \ldots$, and $n_{y}, n_{z}=\ldots,-1,0,1, \ldots$. The noise $\xi$ is expanded in a similar way with

$$
\left\langle\xi_{\mathbf{k}}(t) \xi_{\mathbf{q}}\left(t^{\prime}\right)\right\rangle=\gamma_{k_{x}} V \Gamma \hat{\delta}_{\mathbf{k}, \mathbf{q}} \delta\left(t-t^{\prime}\right)
$$

where $\hat{\delta}_{\mathbf{k}, \mathbf{q}}=\delta_{k_{x}, q_{x}} \delta_{k_{y},-q_{y}} \delta_{k_{z},-q_{z}}$ is a modified three-dimensional Kronecker delta. Moreover, the factor $\gamma_{k_{x}}\left(\gamma_{k_{x}}=1 / 2\right.$ if $k_{x}=0$ and $\gamma_{k_{x}}=1$ otherwise) appears because of the nonflux boundary condition in the $x$ direction. Replacing these expansions in (1), it is found that

$$
\left\langle\phi_{\mathbf{k}}\right\rangle=0, \quad\left\langle\phi_{\mathbf{k}} \phi_{\mathbf{q}}\right\rangle=\gamma_{k_{x}} \hat{\delta}_{\mathbf{k}, \mathbf{q}} V S(\mathbf{k}),
$$

with the same structure factor $S(\mathbf{k})$ as in the homogeneous case. Suppressing again the microscopic self-correlations, the density field fluctuations in real space are given by

$$
\begin{gathered}
\langle\phi(\mathbf{r})\rangle=0, \\
\left\langle\phi(\mathbf{r})^{2}\right\rangle=V^{-1} \sum_{\mathbf{k}}{ }^{\prime} S^{*}(\mathbf{k}) \cos \left(k_{x} x\right)^{2} \\
=\frac{\Gamma}{2 D k_{0}^{2}} V^{-1} \sum_{\mathbf{q}}{ }^{\prime} \frac{1}{q^{2}+1} \cos \left(q_{x} k_{0} x\right)^{2},
\end{gathered}
$$

where $\mathbf{q}=\mathbf{k} / k_{0}$, and the prime in the sums means that the terms $k_{x}=0$ and $q_{x}=0$ have a factor $1 / 2$.

The sum in Eq. (11) contains an ultraviolet divergence $(\mathbf{q} \rightarrow \infty)$. Therefore, in order to perform the summation, a regularization prescription is needed. The divergence is unphysical, because it comes from assuming that the mesoscopic model (1) remains valid up to infinitely large wave vectors. Therefore, we introduce a regularizing kernel in Eq. (11) of the form $1 /\left(1+\epsilon^{2} q^{2}\right)$, which equals 1 for $\epsilon \rightarrow 0$, a limit that will be taken at the end of the calculations. The choice of a rational function instead of an exponential one is made to keep the calculations as simple as possible, but its form is immaterial. The technique of a regularizing kernel is equivalent to imposing a cutoff in the $q$ vectors of the order of $q_{c} \sim \epsilon^{-1}$, or to the $\zeta$ function regularization [29].

Next, we take the limit $L_{y}, L_{z} \rightarrow \infty$, allowing us to replace the sums on $q_{y}$ and $q_{z}$ by integrals that can be carried out, with the result

$$
\left\langle\phi(\mathbf{r})^{2}\right\rangle=\frac{\Gamma}{8 \pi D X} \frac{1}{1-\epsilon^{2}} \sum_{q_{x}}{ }^{\prime} \ln \left(\frac{1+\epsilon^{2} q_{x}^{2}}{\epsilon^{2}\left(1+q_{x}^{2}\right)}\right) \cos \left(q_{x} k_{0} x\right)^{2} .
$$

Figure 1 shows the density fluctuations (12) in the $x$ direction when the plates are located at $x=0$ and $k_{0} L=1 / 2, \epsilon$ $=0.05$, and $L_{x} \rightarrow \infty$. The sets of $q_{x}$ vectors entering in the sum of Eq. (12) for regions I and II are different. In region I, the allowed $q_{x}$ vectors are quantized as $q_{x}=\pi n_{x} /\left(k_{0} L\right)$, while in region II they form a continuum in the limit $L_{x} \rightarrow \infty$. This difference produces a jump of $\left\langle\phi^{2}\right\rangle$ at the plate. 


\section{CASIMIR FORCES}

As shown in Fig. 1, the density fluctuations in a confined system depend on the position but also on the system size, and therefore are different in the region between the plates and the region outside them. As the pressure is a function of the local density field $n$, these differences in the density fluctuations create an unbalance of the pressure at both sides of each plate (see inset of Fig. 1), and consequently a net force.

To calculate the force, we assume that the pressure is a function of the local density, and we expand it around the reference density $n_{0}$. After taking the statistical average, we find that

$$
\langle p(x)\rangle \simeq p\left(n_{0}\right)+\left.\frac{1}{2} \frac{\partial^{2} p}{\partial n^{2}}\right|_{n_{0}}\left\langle\phi(x)^{2}\right\rangle,
$$

where higher-order terms in the field have been neglected.

Therefore the inhomogeneous density fluctuations produce an inhomogeneous pressure profile, similar to those experimentally observed in fluidized granular matter [34]. Note that, when including the regularizing kernel into the sum (11), it turns out that a constant term in $S(\mathbf{k})$ leads to a size-independent contribution to $\left\langle\phi^{2}\right\rangle$ and therefore to the pressure. In consequence, the equilibrium part of the structure factor does not contribute to the Casimir forces, allowing us to eliminate it from the calculations.

The net force acting on the plate located at $x=L$ is the difference between the pressure inside $p\left(x \rightarrow L^{-}\right)$and the pressure outside $p\left(x \rightarrow L^{+}\right)$. These values are calculated from Eq. (12), by replacing the value of $x$ by $L$ [implying that $\left.\cos \left(q_{x} k_{0} L\right)=1\right]$, and using the appropriate set of $q_{x}$ vectors. In the outer part of the plates, region II, the $q_{x}$ vectors form a continuum, and the sum is replaced by an integral:

$$
\left\langle\phi^{2}\right\rangle_{I I}=\frac{\Gamma}{8 \pi D X} \frac{1}{1-\epsilon^{2}} \int d q_{x} \ln \left(\frac{1+\epsilon^{2} q_{x}^{2}}{\epsilon^{2}\left(1+q_{x}^{2}\right)}\right)=\frac{\Gamma}{4 D X} \frac{1}{\epsilon(1+\epsilon)},
$$

which diverges in the limit $\epsilon \rightarrow 0$ as $\mathcal{O}\left(\epsilon^{-1}\right)$. Similarly, in the inner region, we have

$$
\left\langle\phi^{2}\right\rangle_{I}=\frac{\Gamma}{8 \pi D X} \frac{1}{1-\epsilon^{2}} \sum_{q_{x}}{ }^{\prime} \ln \left(\frac{1+\epsilon^{2} q_{x}^{2}}{\epsilon^{2}\left(1+q_{x}^{2}\right)}\right),
$$

with $q_{x}$ forming a discrete set, $q_{x}=\pi n_{x} /\left(k_{0} L\right)$; the summation can be performed with the help of Eq. $(1.431,2)$ of [31]. It also shows a divergence as $\mathcal{O}\left(\epsilon^{-1}\right)$ with the same prefactor as in Eq. (14). Therefore, in the net force between the plates per unit area, obtained as the pressure difference, the two divergent contributions cancel. The resulting force is finite, and, in the limit of a vanishing cutoff $\epsilon$, is simply

$$
F / A=\frac{1}{2} \frac{\partial^{2} p}{\partial n^{2}} \lim _{\epsilon \rightarrow 0}\left[\left\langle\phi^{2}\right\rangle_{I}-\left\langle\phi^{2}\right\rangle_{I I}\right]=F_{0}[1-\ln (2 \sinh l) / l],
$$

where $l=k_{0} L_{x}$ and $F_{0}=\Gamma k_{0}\left(\partial^{2} p / \partial n^{2}\right) /(16 \pi D)$. Let us note that the final expression of the Casimir force, is a universal function of the reduced distance $l=k_{0} L_{x}$. Moreover, there is

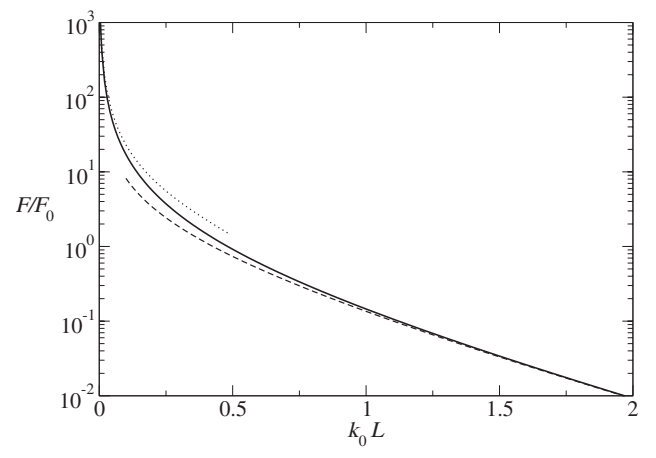

FIG. 2. Dimensionless force as a function of the dimensionless distance $l$, together with the asymptotic form for $l \gg 1$ (dashed line) and $l \ll 1$ (dotted line).

no dependence on the cutoff length, as the two divergences in the cutoff, one stemming from the discrete sum and the other from the integral, exactly cancel each other. The regularizing kernel, a technique well known in the field of Casimir forces [32], has allowed us to obtain a finite result as a difference of two diverging quantities.

The analysis of the Casimir force Eq. (16) can be performed in the limits of far plates $(l \gg 1)$ or near ones $(l \ll 1)$. In the first case, $l \gg 1$ implies that the distance $L_{x} \gg k_{0}^{-1}$, and therefore the plates are outside the correlation length, $k_{0}^{-1}$ [see Eq. (5)]. Then one expects a very fast decay of the Casimir forces. In the opposite limit $(l \ll 1)$, when the plates are well inside the correlation length, the force is much stronger. The expression for these forces are

$$
\frac{F^{f a r}}{A}=F_{0} \frac{e^{-2 l}}{l}, \quad \frac{F^{\text {near }}}{A}=-F_{0} \frac{\log l}{l} .
$$

The expression of $F^{\text {near }}$ is valid only for distances larger than any microscopic cutoff, otherwise the description based on the continuum differential equations (1) becomes meaningless. Therefore no real divergence of the force is obtained for small distances.

In Fig. 2 we plot the exact force Eq. (16) as a function of the dimensionless distance $l$, together with the far and near plate approximations.

\section{SUMMARY AND CONCLUSIONS}

As a summary, we have calculated the fluctuation-induced Casimir force between two parallel plates produced by a scalar field whose evolution is given by a reaction-diffusion equation. This system is out of equilibrium, because the noise terms do not satisfy the fluctuation-dissipation relation, developing long-range correlations. From these correlations, one can calculate the pressure that the fluctuating field exerts on the two parallel plates.

The Casimir force obtained in this way depends on a macroscopic parameter $k_{0}$ that defines a characteristic length as $k_{0}^{-1}$, which is a combination of the diffusion constant and reaction rates, similar to the cases described in other physical systems $[21,28]$. The calculated force exhibits a crossover at plate separations of the order of $k_{0}^{-1}$, a distance at which the 
correlations have decayed [see Eq. (5)]. For short separations, the correlations play an important role, and the force shows a strong distance dependence, while for $L \gg k_{0}^{-1}$ the density correlations have decayed, and the force decays exponentially with the same range $k_{0}^{-1}$ as the correlations.

Finally, the force is repulsive if the pressure is such that $\partial^{2} p / \partial n^{2}>0$, as is usually the case for molecular fluids far from the liquid-gas transition. However, the sign might change for different geometries of the obstacles (for instance, plate and sphere, or two concentric spheres [33]).

To conclude, we have developed a method to calculate Casimir forces starting from the structure factors of the homogeneous system. This method is valid for both equilibrium and nonequilibrium systems, where the usual derivation of Casimir forces based on free-energy calculations is not applicable. The method presented here clarifies the role of the correlation ranges, and their influence over the range of the force. In addition, in comparison with other methods that compute the size-dependent stress tensor, this method is applicable to systems with no scale invariance (that is, with a finite correlation length). The necessary ingredients to obtain a Casimir force are a fluctuating field with correlations that extend over a macroscopic range, and a confinement of the fluctuation spectrum induced by the presence of the plates or other obstacles. The present procedure could be repeated for other systems in nonequilibrium states showing long-range correlations, where it is expected that the resulting Casimir force would depend of the pressure dependence of the fluctuating fields and their dynamics.

\section{ACKNOWLEDGMENTS}

The authors thank J. M. Ortiz de Zárate for useful comments. R.B. acknowledges the hospitality of the Departamento de Física of Universidad de Chile. R.B. is supported by Secretaría de Estado de Educación y Universidades and the Projects MOSAICO, FIS2004-271, and UCM PR27/0513923-BSCH. U.M.B.M. acknowledges COFIN-MIUR Grant No. 2005027808. R.S. acknowledges the hospitality of the Departamento de Física Aplicada I of Universidad Complutense de Madrid. R.S. is supported by Fondecyt research Grants No. 1030993 and No. 1061112 and Fondap Grant No. 11980002.
[1] H. B. G. Casimir, Proc. K. Ned. Akad. Wet. 51, 793 (1948).

[2] M. Kardar and R. Golestanian, Rev. Mod. Phys. 71, 1233 (1999).

[3] M. Fisher and C. R. de Gennes, C. R. Seances Acad. Sci., Ser. B 287, 207 (1978).

[4] H. G. Stanley, Introduction to Phase Transitions and Critical Phenomena (Oxford University Press, Oxford, 1971).

[5] M. Krech, The Casimir Effect in Critical Systems (World Scientific, Singapore, 1994).

[6] I. Brankov, D. M. Danchev, N. S. Tonchev, and J. G. Brankov, Theory of Critical Phenomena in Finite-Size Systems: Scaling and Quantum Effects, Series in Modern Condensed Matter Physics Vol. 9 (World Scientific, Singapore, 2000).

[7] D. Forster, Hydrodynamic Fluctuations, Broken Symmetry, and Correlation Functions (Perseus Books, Boulder, CO, 1995).

[8] A. Ajdari, L. Peliti, and J. Prost, Phys. Rev. Lett. 66, 1481 (1991).

[9] P. G. de Gennes and J. Prost, The Physics of Liquid Crystals (Oxford University Press, Oxford, 1993).

[10] T. Ueno, S. Balibar, T. Mizusaki, F. Caupin, and E. Rolley, Phys. Rev. Lett. 90, 116102 (2003).

[11] A. Martín, A. G. Casielles, M. G. Muñoz, F. Ortega, and R. G. Rubio, Phys. Rev. E 58, 2151 (1998).

[12] J. L. Cardy, in Phase Transitions and Critical Phenomena, edited by C. Domb and J. L. Lebowitz (Academic Press, New York, 1987), Vol. 11.

[13] A. Gambassi and S. Dietrich, J. Stat. Phys. 123, 929 (2006).

[14] D. Dantchev and M. Krech, Phys. Rev. E 69, 046119 (2004).

[15] P. L. Garrido, J. L. Lebowitz, C. Maes, and H. Spohn, Phys. Rev. A 42, 1954 (1990).

[16] J. Machta, I. Oppenheim, and I. Procaccia, Phys. Rev. Lett. 42, 1368 (1979).

[17] J. F. Lutsko and J. W. Dufty, Phys. Rev. E 66, 041206 (2002).
[18] I. Procaccia, D. Ronis, and I. Oppenheim, Phys. Rev. Lett. 42, 287 (1979).

[19] W. B. Li, K. J. Zhang, J. V. Sengers, R. W. Gammon, and J. M. Ortiz de Zárate, Phys. Rev. Lett. 81, 5580 (1998).

[20] J. M. Ortiz de Zárate and J. V. Sengers, Hydrodynamic Fluctuations in Fluids and Fluid Mixtures (Elsevier, Amsterdam, 2006).

[21] H. Spohn, J. Phys. A 16, 4275 (1983).

[22] G. Grinstein, D. H. Lee, and S. Sachdev, Phys. Rev. Lett. 64, 1927 (1990).

[23] I. Pagonabarraga and J. M. Rubí, Phys. Rev. E 49, 267 (1994).

[24] C. W. Gardiner, Handbook of Stochastic Methods (SpringerVerlag, Berlin, 2004).

[25] D. Bartolo, A. Ajdari, and J.-B. Fournier, Phys. Rev. E 67, 061112 (2003).

[26] C. Cattuto, R. Brito, U. M. B. Marconi, F. Nori, and R. Soto, Phys. Rev. Lett. 96, 178001 (2006).

[27] T. P. C. van Noije, M. H. Ernst, E. Trizac, and I. Pagonabarraga, Phys. Rev. E 59, 4326 (1999).

[28] F. van Wijland, K. Oerding, and H. J. Hilhorst, Physica A 251, 179 (1998).

[29] A. Ajdari, B. Duplantier, D. Hone, L. Peliti, and J. Prost, J. Phys. II 2, 487 (1992).

[30] J. García-Ojalvo and J. M. Sancho, Noise in Spatially Extended Systems (Springer-Verlag, New York, 1999).

[31] I. S. Gradshteyn and I. M. Ryzhik, Table of Integrals Series and Products, 5th ed. (Academic Press, New York, 1994).

[32] M. Bordag, U. Mohideen, and V. M. Mostepanenko, Phys. Rep. 353, 1 (2001).

[33] T. H. Boyer, Phys. Rev. 174, 1764 (1968).

[34] S. Aumaitre, C. A. Kruelle, and I. Rehberg, Phys. Rev. E 64, 041305 (2001). 